\title{
The Role of Monitoring the Bcr-Abl Transcript Levels in the Management of Patients with Chronic Myeloid Leukemia
} \author{
Monica1, Pap Zsuzsa ${ }^{3}$ \\ ${ }^{1}$ Clinical Hematology and BMT Unit, County Emergency Clinical Hospital, Tîrgu Mureș, Romania \\ 2 Department of Hematology, University of Medicine and Pharmacy, Tîrgu Mureș, Romania \\ 3 Department of Anatomy, University of Medicine and Pharmacy, Tîrgu Mureș, Romania
}

Tunyogi Aliz-Beáta¹, Benedek I², Benedek Erzsébet¹, Köpeczi Judit Beáta¹, Kakucs Enikő¹, Istrati

\begin{abstract}
Introduction: Chronic myeloid leukemia $(\mathrm{CML})$ is a clonal myeloproliferative disorder; the molecular hallmark of the disease is the $\mathrm{BCR}-\mathrm{ABL}$ gene rearrangement, which usually occurs as the result of a reciprocal translocation between chromosomes 9 and 22. Tyrosine kinase inhibitors (TKI) were the first drugs that targeted the constitutively active BCR-ABL kinase and it have become the standard frontline therapy for $\mathrm{CML}$. Monitoring the treatment of CML patients with detection of bcr-abl transcript levels with real time qualitative polymerase chain reaction (RQ-PCR) is essential in evaluating the therapeutic response.

Material and method: At the Clinical Hematology and BMT Unit Tîrgu Mureș, between 2008-2011, we performed the molecular monitoring of bcr-abl transcript levels with RQ-PCR in 16 patients diagnosed with CML.

Results: We have 11 patients on imatinib treatment who achieved major molecular response. One patient lost the complete molecular response after 5 years of treatment. Two patients in blast crisis underwent allogeneic hematopoietic stem cell transplantation from identical sibling donors. The first patient is in complete molecular remission after 4 years of the transplant with mild chronic GVHD. The other patient had an early relapse with treatment refractory disease and died from evolution of the disease. Three patients with advanced phases of the disease present increasing transcript levels. We performed the dose escalation, and for two of them the switch to the second generation of TKI. Conclusions: Regular molecular monitoring of individual patients with CML is clearly desirable. It allows for a reassessment of the therapeutic strategy in cases of rising levels of BCR-ABL as an early indication of loss of response.
\end{abstract}

Keywords: monitoring CML, RQ-PCR

Received: 3 May 2012

\section{Introduction}

Chronic myeloid leukemia (CML) is a clonal myeloproliferative disorder that arises in the stem cell compartment $[1,2]$. The molecular hallmark of the disease is the BCR-ABL gene rearrangement $[3,4]$, which usually occurs as the result of a reciprocal translocation between chromosomes 9 and 22 [5]. The molecular consequence of this translocation is the generation of a BCR-ABL fusion oncogene, which in turn translates into a $\mathrm{Bcr}-\mathrm{Abl}$ oncoprotein. This most frequently has a molecular weight of $210 \mathrm{kD}(\mathrm{p} 210 \mathrm{Bcr} / \mathrm{Abl})$ and has increased tyrosine kinase activity, which is essential to its transforming capability [6]. Imatinib (previously STI571), a small molecule tyrosine kinase inhibitor (TKI), was the first drug that targeted BCR-ABL and it has become the standard frontline therapy for CML [7]. The majority of patients will do well on standard therapy. However, some patients will fail on imatinib and require alternative therapies. Currently, the main tool to identify high-risk patients is close monitoring of their in vivo response to therapy [8]. Realtime quantitative polymerase chain reaction (RQ-PCR)

Correspondence to: Aliz-Beáta Tunyogi

E-mail: tunyogializ@yahoo.co.uk provides an accurate measure of the total leukemia cell mass and the degree to which BCR-ABL transcripts are reduced by therapy correlates with progression-free survival [9]. If they do not achieve a complete hematologic response with 3 months of imatinib, if they are predominantly $\mathrm{PH}$ positive at 6 months or still have $>35 \% \mathrm{PH}$ positive metaphases at 12 months, or the loss of a previous hematologic or cytogenetic response, or had a $1 \log$ increase in BCR-ABL transcripts in patients who had achieved a complete cytogenetic response, is defined as failure to imatinib therapy [10]. Identification of those patients who are likely to fail is desirable to allow for more intensive therapy up front [8].

\section{Material and methods}

At the Clinical Hematology and BMT Unit Tîrgu Mureș, between 2008-2011 we performed the molecular monitoring of bcr-abl transcript levels with RQ-PCR in 16 patients diagnosed with CML. We collected a complete set of baseline data: a clinical examination with documentation of spleen size, complete blood count (CBC) with white blood cell differential, and bone marrow biopsy with metaphase karyotyping, RQ- PCR for BCR-ABL rearrangement. For the patients in advanced phase of the disease, flowcyto- 
SK, CML in blastic transformation, $41 \%$ blasts
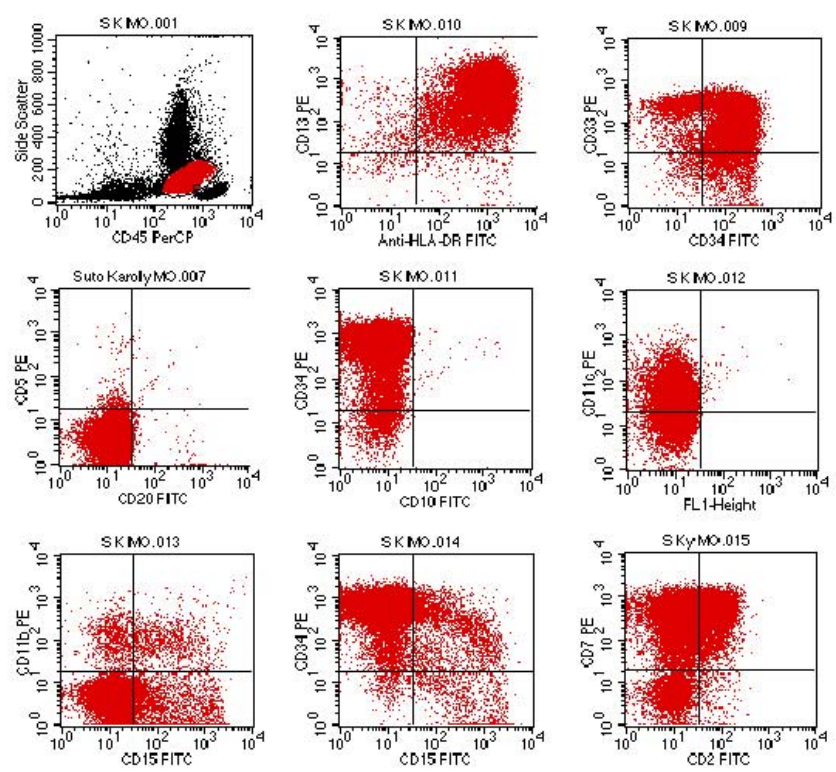

Fig. 1. Case 2: Flow cytometry before transplantation

metry was performed from bone marrow to determine the percent and the phenotype of the blast cells.

Due to the small size of the sample of patients, statistic analysis seemed to be inappropriate.

\section{Results}

The median age at diagnosis was 44 years. The majority of the patients were in first chronic phase at diagnosis (10 patients, 62\%), 3 patients (19\%) in accelerated phase and 3 patients (19\%) in blast crisis. The Sokal score was calculated and 15 patients were identified as high risk patients, while one patient presented intermediary prognostic score.

The $1^{\text {st }}$ patient is a 48 year old female, diagnosed with CML in 1996, first treated with hydroxiureea and then with imatinib $400 \mathrm{mg} /$ day. In 2006, under the imatinib treatment, the disease progressed to accelerated phase (bcrabl transcript levels 25.8\%).

The patient's dose was escalated to imatinib $600 \mathrm{mg} /$ day, but she presented blastic transformation ( $80 \%$ blasts in peripheral blood). In March 2008 we performed the allogeneic stem cell transplantation from an HLA identical sibling. Immunosuppression was performed with Cyclosporine + Methotrexate. She presents mild chronic GVHD under low

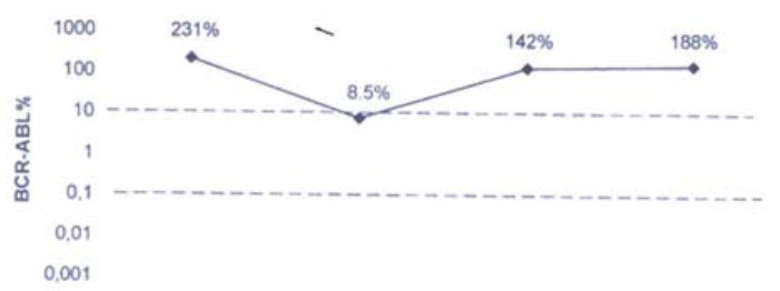

Fig. 3. Case 2: Modification of the bcr-abl transcript levels during the treatment
SK, PB, $80 \%$ blasts
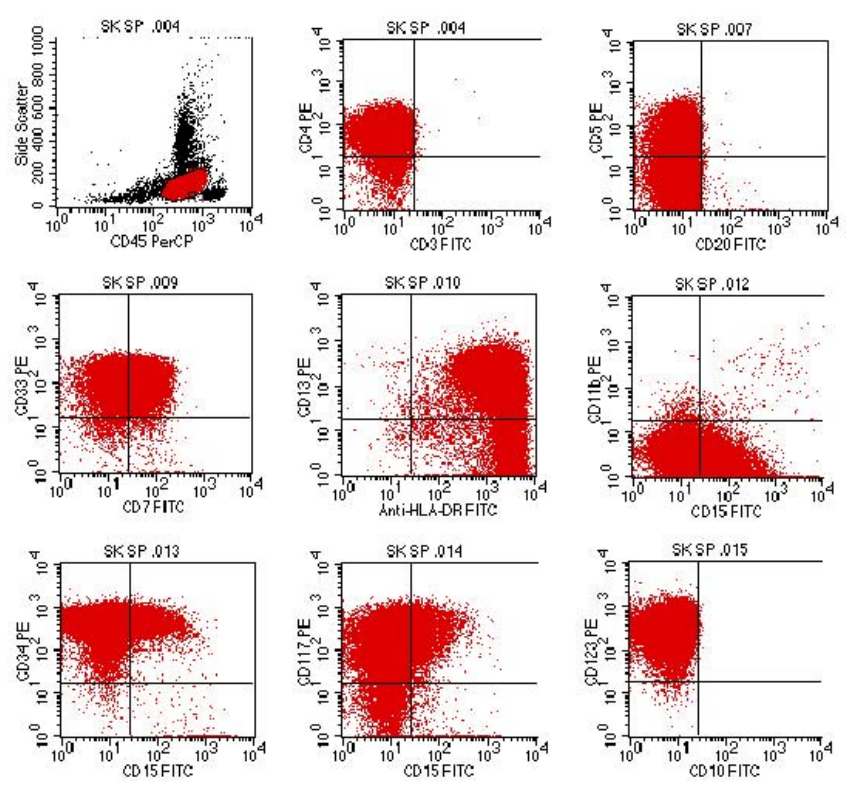

Fig. 2. Case 2: Flowcytometry performed in May 2009, 8 months following the transplantation

dose immunosuppression (Medrol $4 \mathrm{mg}$ ). She is in complete molecular remission 4 years after the transplantation.

The $2^{\text {nd }}$ patient is a 41 year old male, diagnosed in 2007. Under imatinib treatment $400 \mathrm{mg} /$ day he presented blastic transformation of the disease (flowcytometry performed).

In September 2008 we performed the allogeneic stem cell transplantation from an HLA identical sibling donor. Immunosuppression was performed with Cyclosporine + Methotrexate. The patient did not present any signs of GVHD. Short time after the transplantation an early relapse occurred. We stopped the immunosuppression and initiated Imatinib $600 \mathrm{mg} /$ day and donor lymphocyte infusion (DLI), with no response. In January 2009 the flowcytometry revealed $30 \%$ of blasts in peripheral blood and bcr-abl levels were $142 \%$. We initiated treatment with high dose chemotherapy and dasatinib $2 \times 70 \mathrm{mg} /$ day. In May 2009 the peripheral blast count (80\%) and the bcrabl transcript levels were increasing (188\%).

We switched the dasatinib to nilotinib $2 \times 400 \mathrm{mg} /$ day.

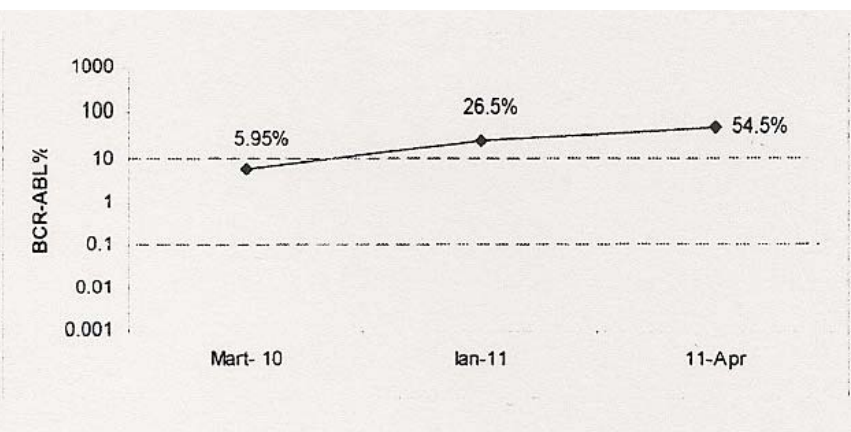

Fig. 4. Case 3. Modification of the bcr-abl transcript levels during the treatment 


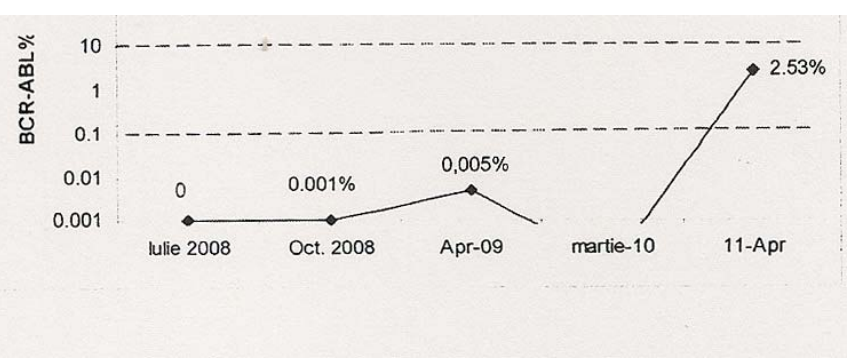

Fig. 5. Case 6. Modification of the bcr-abl transcript levels during the treatment

A year after transplantation and HD chemotherapy the patient presented left lobar pneumonia, septicemia and died due to multiorgan failure.

The $3^{\text {rd }}$ case was in accelerated phase of the disease at presentation, received imatinib $400 \mathrm{mg} / \mathrm{day}$, but the levels of bcr-abl transcripts increased and the patient's dose was escalated to Imatinib $600 \mathrm{mg} /$ day.

The $4^{\text {th }}$ case was under treatment with hydroxiureea presenting blastic transformation, bcr-abl transcript levels were $167.3 \%$. The patient received induction chemotherapy and imatinib $600 \mathrm{mg} /$ day. The patient went to the second chronic phase (bcr-abl transcript levels 24.9\%). After four months under imatinib the patient presented $90 \%$ of blasts and increasing transcript levels (199\%). Under high dose chemotherapy + dasatinib $2 \times 70 \mathrm{mg} /$ day the transcript levels decreased (75\%).

In the $5^{\text {th }}$ case, with blast crisis on presentation, bcr-abl transcript levels were $34 \%$. We initiated induction chemotherapy and imatinib $600 \mathrm{mg} /$ day. The transcript levels increased $(42 \%)$ and we switched to the second generation of TKIs - dasatinib $2 \times 70 \mathrm{mg} /$ day and then nilotinib $2 \times 400$ $\mathrm{mg} /$ day, with slightly decreasing transcript levels $(28 \%)$, but no hematological response.

In the $6^{\text {th }}$ case we witnessed a loss of complete molecular response after 5 years of imatinib treatment $400 \mathrm{mg} /$ day. The switch to the second generation of TKI the mutational analysis and cytogenetic reevaluation are indicated.

We have 10 patients on imatinib $400 \mathrm{mg} / \mathrm{day}, 3$ of them in accelerated phase and 7 in chronic phase at diagnosis, who achieved major molecular response, and four of them completed molecular response with no detectable transcript levels. The median follow-up time was 5 years and 6 months.

\section{Discussion}

With the introduction of TKIs in CML therapy, the outstanding therapeutic results have shifted the attention to molecular studies. Patients with Philadelphia chromosome $(\mathrm{Ph})$-positive CML who are in complete cytogenetic response (CCyR) on tyrosine kinase inhibitors (TKIs), are frequently monitored for response by RQ-PCR for minimal molecular disease, to identify which patients in CCyR would have a high likelihood of relapse, and who might therefore benefit from escalating the imatinib dose or changing therapy to the more potent second generation TKIs or allogeneic stem cell transplantation. In the first

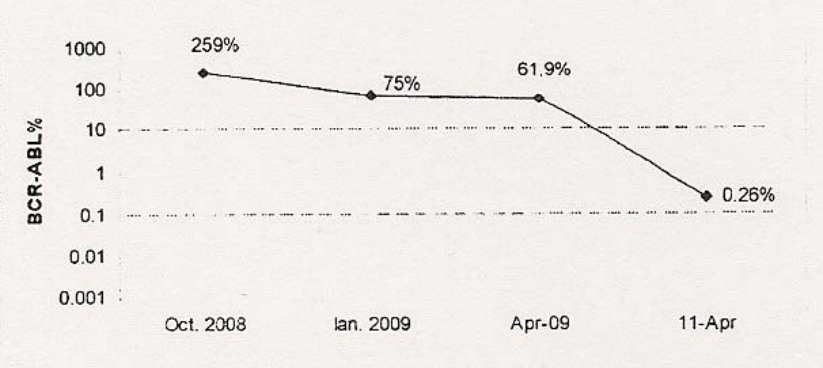

Fig. 6. Case 7. Modification of the bcr-abl transcript levels during the treatment

two patients with identical sibling donors the increasing transcript levels under imatinib helped us to decide for allogeneic stem cell transplantation. In the second case the increasing transcript levels determined us to stop the immunosuppression and administer donor lymphocyte infusion before the hematological changes appeared and guided our decision of switch between second generations of TKI. In three cases the increasing transcript levels and the absence of hematological response determined us to initiate treatment with second generation TKI.

In our lot $68.5 \%$ of patients achieved major molecular response. Among these patients only two were in accelerated phase of the disease, the others presented in the first chronic phase. Responses are durable in most patients treated in early chronic phase, particularly among those who achieve major molecular responses [6]. The median time for follow-up was 5 years and 6 months and we witnessed the loss of complete molecular response in only one patient. Patients who lose MMR or never achieve MMR, and have more than $1 \log$ increase of RQ-PCR, should be monitored more closely, and may be evaluated for mutations of BCR-ABL kinase domain and considered for investigational therapeutic interventions [11].

\section{Conclusions}

- The RQ-PCR is a proven method for the monitoring of $\mathrm{Ph}$-positive CML patients who are in complete cytogenetic response (CCyR) on tyrosine kinase inhibitors, for the evaluation of therapeutic response.

- Increasing transcriptional levels of BCR-ABL is an early indicator of the loss of therapeutic response and could provide clinical benefits by triggering early therapeutic interventions.

- RQ-PCR provides an accurate measure of the total leukemic cell mass. In those patients who achieved major molecular response on TKIs the response is durable and is associated in our study with progression-free survival.

- Patients who lose MMR or never achieve MMR, and have more than $1 \log$ increase of RQ-PCR, should be monitored more closely.

\section{References}

1. Copland M. Chronic myelogenous leukemia stem cells: What's new? Curr Hematol Malig Rep. 2009;4(2):66-73. 
2. Deininger M, Goldman J, Melo J. The molecular biology of chronic myeloid leukemia. Blood. 2000;96:3343-3356.

3. Serpa M, Sanabani SS, Dorliac-Llacer PE, Conchon M. Molecular measurement of BCR-ABL transcript variations in chronic myeloid leukemia patients in cytogenetic remission. BMC Blood Disorders. 2010;10:7.

4. Pavlu J, Richard MSz, Goldman JM, Apperley JF. Three decades of transplantation for chronic myeloid leukemia: what have we learned? Blood. 2011;117:755-763.

5. Belli C, Alú MF, Alfonso G, Bianchini M, Larripa I. Novel variant Ph translocation $\mathrm{t}(9 ; 22 ; 11)(q 34 ; q 11.2 ; p 15)$ inv(9)(p13q34) in chronic myeloid leukemia involving a one-step mechanism. Cytogenet Genome Res. 2011;132(4):304-8. Epub 2011 Jan 6.

6. Jabbour E, Cortes J, Kantarjian H. Optimal First-Line Treatment of Chronic Myeloid Leukemia: How to Use Imatinib and What Role for Newer Drugs? Oncology. 2007;21(6):653-62.
7. Litzow MR. Imatinib resistance: obstacles and opportunities. Arch Pathol Lab Med. 2006;130:669-679.

8. Deininger MW. Milestones and Monitoring in Patients with CMLTreated with Imatinib. ASH Education Book. 2008;1:419-426.

9. Hughes T, Deininger M, Hochhaus A, et al. Monitoring CML patients responding to treatment with tyrosine kinase inhibitors: review and recommendations for harmonizing current methodology for detecting $\mathrm{BCR}-\mathrm{ABL}$ transcripts and kinase domain mutations and for expressing results. Blood. 2006;108(1):28-37.

10. Provan D, Singer RJ, Baglin T, Dokal I. Oxford Handbook of Clinical Hematology, Oxford University Press, third edition, Oxford, 2009, pg. 140-147.

11. Kantariian HM, Shan J, Jones D, et al. Significance of Increasing Levels of Minimal Residual Disease in Patients With Philadelphia ChromosomePositive Chronic Myelogenous Leukemia in Complete Cytogenetic Response J Clin Oncol. 2009;27(22):3659-3663. 